\title{
Safety and efficacy of endoscopic spray cryotherapy for Barrett's esophagus with high-grade dysplasia
}

\author{
Nicholas J. Shaheen, MD, MPH, Bruce D. Greenwald, MD, Anne F. Peery, MD, John A. \\ Dumot, MD, Norman S. Nishioka, MD, Herbert C. Wolfsen, MD, J. Steven Burdick, MD, \\ Julian A. Abrams, MD, Kenneth K. Wang, MD, Damien Mallat, MD, Mark H. Johnston, MD, \\ Alvin M. Zfass, MD, Jenny O. Smith, MD, James S. Barthel, MD, and Charles J. Lightdale, \\ MD \\ Chapel Hill, North Carolina, Baltimore, Maryland, Cleveland, Ohio, Jacksonville, Florida, Dallas, \\ Texas, New York, New York, Rochester, Minnesota, Lancaster, Pennsylvania, Richmond, \\ Virginia, Tampa, Florida, USA
}

\begin{abstract}
Background-Endoscopic ablation to treat Barrett's esophagus (BE) with high-grade dysplasia (HGD) is associated with a decreased incidence of esophageal adenocarcinoma. Endoscopic spray cryotherapy (CRYO) demonstrates promising preliminary data.
\end{abstract}

Objective-To assess the safety and efficacy of CRYO in BE with HGD.

Design-Multicenter, retrospective cohort study.

Setting-Nine academic and community centers; treatment period, 2007 to 2009.

Patients-Subjects with HGD confirmed by 2 pathologists. Previous EMR was allowed if residual HGD remained.

Interventions-CRYO with follow-up biopsies.

Main Outcome Measurements-Complete eradication of HGD with persistent low-grade dysplasia, complete eradication of all dysplasia with persistent nondysplastic intestinal metaplasia, and complete eradication of all intestinal metaplasia.

Results-Ninety-eight subjects (mean age 65.4 years, 83\% male) with BE and HGD (mean length $5.3 \mathrm{~cm}$ ) underwent 333 treatments (mean 3.4 treatments per subject). There were no esophageal perforations. Strictures developed in 3 subjects. Two subjects reported severe chest pain managed with oral narcotics. One subject was hospitalized for bright red blood per rectum. Sixty subjects had completed all planned CRYO treatments and were included in the efficacy analysis. Fifty-eight subjects (97\%) had complete eradication of HGD, 52 (87\%) had complete eradication of all dysplasia with persistent nondysplastic intestinal metaplasia, and 34 (57\%) had complete eradication of all intestinal metaplasia. Subsquamous BE was found in 2 subjects (3\%).

Copyright @ 2010 by the American Society for Gastrointestinal Endoscopy

Reprint requests: Nicholas J. Shaheen, MD, MPH, Center for Esophageal Diseases and Swallowing, University of North Carolina School of Medicine, CB\#7080, Chapel Hill, NC 27599-7080..

Current affiliations: Center for Esophageal Diseases and Swallowing (N.J.S., A.F.P.), University of North Carolina School of Medicine, Chapel Hill, North Carolina, Division of Gastroenterology and Hepatology (B.D.G.), University of Maryland School of Medicine and Greenebaum Cancer Center, Baltimore, Maryland, Department of Gastroenterology and Hepatology (J.A.D.), Cleveland Clinic, Cleveland, Ohio, Massachusetts General Hospital, Harvard Medical School (N.S.N.), Boston, Massachusetts, Mayo Clinic Jacksonville (H.C.W.), Jacksonville, Florida, Texas Digestive Health Associates (J.S.B.), Dallas, Texas, Columbia University Medical Center (J.A.A., C.J.L.), New York, New York, Mayo Clinic Rochester (K.K.W.), Rochester, Minnesota, Digestive Health Associates of Texas (D.M.), Dallas, Texas, Lancaster Gastroenterology Inc (M.H.J.), Lancaster, Pennsylvania, Virginia Commonwealth University (A.M.Z., J.O.S.), Richmond, Virginia, and Moffitt Cancer Center (J.S.B.), Tampa, Florida. 
Limitations-Nonrandomized, retrospective study with no control group, short follow-up (10.5 months), lack of centralized pathology, and use of surrogate outcome for decreased cancer risk.

Conclusions-CRYO is a safe and well-tolerated therapy for BE and HGD. Short-term results suggest that CRYO is highly effective in eradicating HGD.

Barrett's esophagus (BE) is a metaplastic change in the lining of the esophagus from the normal squamous lining to specialized columnar epithelium. This change, thought to be caused by injury from chronic reflux disease, is associated with a 40- to 120 -fold increase in the risk of esophageal adenocarcinoma. ${ }^{1,2}$ When BE does progress, it seems to do so through a series of changes from nondysplastic to low-grade dysplasia (LGD) to high-grade dysplasia (HGD) to cancer. Although the risk of cancer in patients with nondysplastic disease and LGD seems to be less than $1 \%$ per patient-year, ${ }^{3,4}$ that risk increases substantially with $\mathrm{HGD},{ }^{5,6}$ making an intervention desirable to a large proportion of patients.

Surgical esophagectomy, the traditional intervention offered to subjects with BE and HGD, is associated with substantial morbidity and some mortality. ${ }^{7}$ Additionally, because most subjects diagnosed with HGD are found to have the condition later in life, many are not surgical candidates. Therefore, investigators have developed a variety of endoscopic interventions designed to eradicate HGD and induce reversion to squamous epithelium while leaving the esophagus intact. All of these therapies take advantage of the seminal observation that rigorous acid suppression combined with BE cell destruction usually results in partial or full regeneration of squamous epithelium. ${ }^{8}$ Successful regeneration of squamous epithelium seems to be associated with a decreased risk of adenocarcinoma of the esophagus. ${ }^{5,6}$ Endoscopic therapy may be preceded by EMR of nodular epithelium. Alternatively, stepwise radical mucosal resection may be used to remove all metaplastic tissue. $^{9}$

Endoscopic spray cryotherapy (CRYO) using liquid nitrogen is a novel method of destruction of BE tissue. Initial reports suggest that this therapy is well tolerated and effective in inducing neosquamous epithelium in subjects with BE. ${ }^{10,11}$ This retrospective study reports the safety and efficacy of CRYO in subjects with BE and HGD.

\section{METHODS}

\section{Study design and subjects}

We performed a retrospective analysis of consecutive subjects with BE and HGD undergoing CRYO at 9 sites in the United States. Subjects with unifocal or multifocal HGD of any length were eligible. All pathological specimens demonstrating HGD were confirmed with a second histological analysis by an expert pathologist. Previous EMR or other previous endoscopic therapy was allowed if the results demonstrated no evidence of residual adenocarcinoma and residual HGD was present in the tubular esophagus at commencement of CRYO. CRYO was performed with the intent to eradicate all BE. Each site participating in the study offers comprehensive therapy for BE. All subjects signed informed consent for the endoscopic procedures, and the protocol for retrospective, anonymous collection of these data was reviewed and approved by the institutional review boards of all participating centers.

\section{Take-Home Message}

- Multiple methods have been described to perform endoscopic ablative therapy in the setting of Barrett's esophagus. Endoscopic cryotherapy, in this 
preliminary report, seems safe and efficacious in downgrading dysplasia in subjects with Barrett's esophagus and high-grade dysplasia.

\section{Study intervention}

Subjects were sedated by using either propofol (23\% of procedures) or a combination of narcotic and benzodiazepine (77\% of procedures). Standard diagnostic upper endoscopy was performed. Subjects were then treated with the CSA cryotherapy system (CSA Medical, Baltimore, Md). This system is a noncontact method of cryotherapy, applying medical-grade $-196^{\circ} \mathrm{C}$ liquid nitrogen by using a low-pressure, disposable $7 \mathrm{~F}$ spray catheter directly to the tissue targeted for treatment at a cooling rate of $25 \mathrm{~W}$. The system enables the physician to control the duration of the cryogen spray to the selected site by depressing a foot pedal.

Before the administration of the liquid nitrogen, a duallumen decompression tube was placed in the gastric antrum to evacuate nitrogen gas by both passive venting and active suction (Fig. 1A). To administer the liquid nitrogen, a polyamide catheter was introduced through the working channel of an adult endoscope and positioned 3 to $4 \mathrm{~mm}$ beyond the distal tip of the endoscope (or at the distal edge of a clear, friction-fit cap, if used). Gas flow was initiated by depressing a foot pedal. The treatment area was limited to 2 to $3 \mathrm{~cm}$ of mucosa in a hemicircumferential or one third circumferential pattern so that freezing could be maintained without reperfusion during the treatment. In situations in which the decompression tube blocked the flow of cryogen onto selected sites, the endoscope tip was used to gently reposition the tube out of the path of the spray. The endoscopist monitored the endoscopic appearance of the tissue, and when white frost was noted in the treatment area, a timer was actuated to track the freeze time (Fig. 1B). Treatment dosimetry (the duration of the tissue freeze time in seconds and the number of cycles in which the target site was frozen and thawed) was either 2 cycles of 20 seconds or 4 cycles of 10 seconds. During the intervening thaw cycles, the tissue reperfused, with return to its normal salmon color. Monitoring of the abdomen was performed by a nurse or technician for any signs of abdominal distention. Typical initial treatments involved 3 to 5 treatment sites.

All procedures were performed on an outpatient basis. Subjects were discharged with acetaminophen for pain control. Follow-up procedures were performed at 2- to 3-month intervals until eradication of $\mathrm{BE}$ was confirmed by both histological and endoscopic findings or until the clinician and/or patient opted to halt treatment for clinical reasons, including worsening comorbidities, disease progression, and patient preference.

\section{Biopsy technique and histological analysis}

At follow-up endoscopy sessions, biopsies were performed every 1 to $2 \mathrm{~cm}$ in 4 quadrants. Each endoscopic biopsy level specimen was placed in a separate container for analysis. Tissue was fixed in formalin and subsequently embedded in paraffin. Tissue was stained with hematoxylin and eosin. Biopsy specimens were interpreted at the home institution by pathologists expert in the assessment of BE. Any reading of HGD or cancer was confirmed by a second pathologist. Biopsy specimens were graded by using standardized criteria for $\mathrm{BE}$, requiring the presence of goblet cells for the diagnosis. ${ }^{12}$

\section{Data collection and analysis}

All participating sites use electronic medical records. For each subject, data of the following variables were collected: age, sex, initial length of the BE, EMR history, any complications of the ablative therapy, and the results of the ablative therapy. The potential results of ablative therapy included progression to esophageal adenocarcinoma, continued presence of HGD, complete eradication of HGD with persistent LGD, eradication of all dysplasia with 
persistent nondysplastic intestinal metaplasia, and eradication of all intestinal metaplasia. Potential complications assessed included perforation, stricture requiring dilation, bleeding requiring transfusion, and pain requiring narcotic analgesia or hospitalization. Stricture was defined as an endoscopically evident narrowing of the esophagus, with or without the patient reporting dysphagia. Additionally, for each diagnostic or therapeutic procedure, we recorded the total procedure time, sedation used, performance of any endoscopic biopsies, and histology results.

For purposes of this analysis, we divided the cohort into 2 groups. For the safety analysis, all subjects and all treatments were analyzed. For the efficacy analysis, we included subjects who had at least 1 postablation endoscopy with biopsies and for whom no further CRYO treatment was planned.

For continuous variables such as age and medication dose, mean and standard deviation (SD) are reported. For categorical data, proportions are reported. To compare responders with nonresponders, the Fisher exact and $\chi^{2}$ tests were used. All tests of significance were 2 tailed, and $\alpha$ values $<.05$ were considered significant.

\section{RESULTS}

A total of 98 subjects fulfilled criteria and were included in the study. Mean (SD) age was 65.4 (10) years, and $83 \%$ were male (Table 1). The baseline mean (SD) endoscopic length of $\mathrm{BE}$ was $5.3(3.2) \mathrm{cm}$. Before enrollment in the study, 1 (1\%) subject had undergone Nissen fundoplication. Two (2\%) subjects were status post partial esophagectomy for HGD with recurrent HGD in the esophageal remnant. Fourteen (14\%) subjects were status post some form of previous unsuccessful ablative therapy ( $2 \%$ argon plasma coagulation, $6 \%$ radiofrequency ablation [RFA], 6\% photodynamic therapy [PDT]), and $22(22 \%)$ subjects were status post EMR. EUS was performed on $71 \%$ of subjects before therapy to assess for evidence of locoregional or celiac lymphadenopathy.

Of the 98 subjects enrolled in the study, 60 had completed all planned CRYO treatments, with the remainder still undergoing planned therapy. The baseline characteristics of the subjects who had completed therapy did not differ significantly from those still undergoing therapy (Table 1).

\section{Efficacy analysis}

Figure 2 demonstrates the efficacy results of the study. Of the 60 patients who completed all planned CRYO treatments, 58 (97\%) had complete eradication of HGD, 52 (87\%) had complete eradication of intestinal dysplasia, and 34 (57\%) had complete eradication of intestinal metaplasia. These subjects underwent a mean (SD) of $4.0(2.9)$ treatments.

Subjects completing CRYO treatments have been followed for a mean (SD) of 10.5 (8.3) months after completing CRYO with 3- to 6-month surveillance endoscopies per the institutions' postablation protocols. Subsquamous BE was found on biopsy specimens in 2 (3\%) subjects (Fig. 3).

\section{Safety analysis}

A total of 333 treatments were performed in the 98 subjects enrolled in the study (mean 3.4 treatments per subject), all on an outpatient basis (Table 2). There were no serious adverse events or perforations. Strictures (3\% of patients, $1 \%$ of treatments) developed in 3 subjects, and all underwent successful endoscopic dilation (2, 3, and 3 dilations, respectively, for symptom resolution). Two subjects reported severe chest discomfort after the procedure ( $2 \%$ of patients, $1 \%$ of treatments) and were successfully managed with oral narcotics on an 
outpatient basis. There was disease progression in 1 patient despite treatment. In this patient, a nodule was noted at the gastroesophageal junction at the second planned CRYO treatment. EMR was performed, with pathology findings of intramucosal adenocarcinoma. This subject underwent successful esophagectomy and is cancer free 1 year later. One subject required hospitalization post-procedure for bright red blood per rectum. This subject was admitted and observed overnight with no significant change in hematocrit or additional evaluation performed.

\section{DISCUSSION}

Endoscopic ablation to eradicate HGD and induce reversion to squamous epithelium is a viable, esophagus-sparing treatment option for patients with BE and HGD. Multiple methods of endoscopic ablation have been developed. Each method differs in mechanism and effectiveness of ablation, side effects, and cost. Our retrospective analysis of subjects with HGD treated with CRYO at 9 institutions, the largest reported experience with this technique to date, suggests that CRYO is associated with a favorable side effect profile and leads to reversion to squamous epithelium in the majority of treated subjects. We found that the therapy was well tolerated, with only 1 hospitalization after 333 treatments and a stricture rate of $3 \%$.

Previous data on endoscopic cryotherapy using either liquid nitrogen or carbon dioxide suggest that the treatment is effective in downgrading dysplasia and causing reversion of intestinal metaplasia to neosquamous epithelium. In 2005 , Johnston et al ${ }^{10}$ reported a pilot single-center study of CRYO in 11 subjects with metaplasia and/or dysplasia. Nine subjects completed the study, and all had complete eradication of both metaplasia and dysplasia at the end of treatment. Therapy was well tolerated, and there were no reports of perforations, strictures, or severe chest pain. In 2008, Canto et al ${ }^{13}$ reported in abstract form a singlecenter experience of carbon dioxide spray cryotherapy in 33 subjects with HGD and/or intramucosal carcinoma. EMR and/or PDT failed in all subjects, and they were considered at high risk of esophagectomy. At the time that the data were reported, the study was ongoing and a mean of 3 treatments per subject had been performed. They reported a preliminary $72 \%$ mean reduction in BE in subjects still undergoing treatment. Therapy was well tolerated, and there were no reports of perforations, strictures, or severe chest pain.

In 2009, Dumot et $\mathrm{al}^{14}$ reported a single-center study of CRYO in 30 subjects with HGD and/or intramucosal carcinoma. All subjects were considered at high risk of esophagectomy or had refused surgical intervention. They found that $90 \%$ of the subjects treated had a downgrading of pathology stage. In this study, 3 (10\%) subjects reported severe chest pain and $3(10 \%)$ had strictures requiring dilation. Also, in this series, a gastric perforation developed during treatment as a result of excessive gastric distention in a patient with Marfan syndrome. Concerns of barotrauma of the stomach resulted in the development of a dual-lumen orogastric decompression study, with both active and passive venting of gas to prevent overdistention. These data were also included in a multicenter study of CRYO by Greenwald et al. ${ }^{11}$ In this 4-center study, 77 subjects with metaplasia, LGD, HGD, intramucosal carcinoma, invasive carcinoma, or severe squamous dysplasia were treated with CRYO therapy. Of the 17 subjects with HGD who had completed therapy, $94 \%$ had complete eradication of HGD, 88\% had complete eradication of dysplasia, and $53 \%$ had complete eradication of intestinal metaplasia. Overall, a total of 323 treatments were performed in the cohort, with strictures requiring dilation developing in 3 subjects and $3.7 \%$ reporting moderate or severe chest discomfort.

Given the preliminary nature of the data regarding the efficacy of CRYO in the treatment of dysplastic BE, it is challenging currently to compare this modality with other available 
choices for ablative therapy. Our preliminary safety data compare favorably with those of PDT. Our stricture rate is far lower than that reported in case series ${ }^{15}$ and in the randomized, controlled trial of PDT versus medical therapy performed by Overholt et al. ${ }^{6}$ Additionally, rates for complete clearance of intestinal metaplasia in this study are higher than those reported with PDT. ${ }^{6}$ How does CRYO compare with RFA? RFA has an excellent safety profile, similar to that found in this study. ${ }^{5,16}$ Although the $74 \%$ rate of complete eradication of intestinal metaplasia ( $82 \%$ per protocol) reported in the HGD arm of a recent RFA shamcontrolled, randomized trial ${ }^{5}$ is higher than the $57 \%$ rate reported here, the subjects in the RFA trial all had BE of less than $8 \mathrm{~cm}$, were treatment naïve, and fulfilled other exclusion criteria not present in the current study. Ganz et al ${ }^{17}$ reported outcomes of 142 subjects with HGD treated with RFA as part of the RFA HGD registry. This cohort is most comparable to that in the present study because it was retrospective in nature, had few exclusion criteria, and was collected from multiple centers. Interestingly, in this registry, complete eradication of intestinal metaplasia occurred in 54\% of subjects, a figure virtually identical to the $57 \%$ reported here. One potential advantage to CRYO is that tissue apposition to a probe or device is not needed for efficacy. Therefore, such a spray application might have special utility when firm tissue contact is either unattainable or made difficult because of previous endoscopic therapy such as EMR.

The strengths of our study include an analysis of data obtained from both community and academic centers and the inclusion of a large number of patients with HGD. Study definitions were standardized, and rigorous, histology-based outcomes were used. Our study also had several limitations. We used a retrospective cohort with short-term follow-up (10.5 months), which is subject to bias. The effect of ablative therapy can be exaggerated in observational studies by the combined factors of spontaneous regression, sampling error, and variability of histological interpretation of biopsy specimens. ${ }^{18}$ Although all the participating centers are expert in the care of subjects with BE, pathology from the centers was not reviewed by a central laboratory. Our primary outcome in this study, eradication of intestinal dysplasia and metaplasia, was used as a surrogate outcome marker for decreased cancer risk, and data linking the two are sparse. ${ }^{5,6}$ As with all ablative therapies that do not recover a specimen for histological evaluation, the potential for understaging an invasive adenocarcinoma exists. Although the risk of submucosal cancer seems low in appropriately chosen subjects with HGD, ${ }^{9}$ better definition of this risk is necessary.

In conclusion, CRYO is a safe and well-tolerated therapy for subjects with BE and HGD. Short-term results suggest that the therapy is effective in eradicating HGD and that a majority of subjects will have complete eradication of all intestinal metaplasia as well. Further analysis of this cohort will determine the durability of the changes reported here as well as the cancer risk in subjects treated with this modality.

\section{Acknowledgments}

The following authors disclosed financial relationships relevant to this publication: N.J. Shaheen: Grant from, consultant for, and advisory committee member of CSA Medical; grant from BÂRRX Medical Inc. B.D. Greenwald: Consultant for and advisory committee member of CSA Medical. J.A. Dumot: Consultant for and advisory committee member of CSA Medical. N.S. Nishioka: Research support from CSA Medical. H.C. Wolfsen: Research support from CSA Medical; research support from BÂRRX Medical Inc. K.K. Wang: Research support from CSA Medical; research support from BÂRRX Medical Inc. M.H. Johnston: Research support from CSA Medical. J.S. Barthel: Research support from CSA Medical. C.J. Lightdale: Advisory committee member of CSA Medical. All other authors disclosed no financial relationships relevant to this publication. This study was funded in part with a grant from CSA Medical, makers of the spray cryotherapy device. Dr. Peery is supported by a grant from the National Institutes of Health (T32 DK 07634). Statistical analysis and data management were also supported by a grant from the National Institutes of Health (P30 DK034987). Dr. Abrams is supported in part by a K07 award from the National Cancer Institute (CA 132892). 


\section{Abbreviations}

$\begin{array}{ll}\text { BE } & \text { Barrett's esophagus } \\ \text { CRYO } & \text { endoscopic spray cryotherapy } \\ \text { HGD } & \text { high-grade dysplasia } \\ \text { LGD } & \text { low-grade dysplasia } \\ \text { PDT } & \text { photodynamic therapy } \\ \text { RFA } & \text { radiofrequency ablation } \\ \text { SD } & \text { standard deviation }\end{array}$

\section{REFERENCES}

1. Shaheen NJ, Richter JE. Barrett's oesophagus. Lancet. 2009; 373:850-61. [PubMed: 19269522]

2. Spechler SJ. Clinical practice. Barrett's esophagus. N Engl J Med. 2002; 346:836-42. [PubMed: 11893796]

3. O'Connor JB, Falk GW, Richter JE. The incidence of adenocarcinoma and dysplasia in Barrett's esophagus: report on the Cleveland Clinic Barrett's Esophagus Registry. Am J Gastroenterol. 1999; 94:2037-42. [PubMed: 10445525]

4. Drewitz DJ, Sampliner RE, Garewal HS. The incidence of adenocarcinoma in Barrett's esophagus: a prospective study of 170 patients followed 4.8 years. Am J Gastroenterol. 1997; 92:212-5. [PubMed: 9040193]

5. Shaheen NJ, Sharma P, Overholt BF, et al. Radiofrequency ablation in Barrett's esophagus with dysplasia. N Engl J Med. 2009; 360:2277-88. [PubMed: 19474425]

6. Overholt BF, Lightdale CJ, Wang KK, et al. Photodynamic therapy with porfimer sodium for ablation of high-grade dysplasia in Barrett's esophagus: international, partially blinded, randomized phase III trial. Gastrointest Endosc. 2005; 62:488-98. [PubMed: 16185958]

7. Birkmeyer JD, Siewers AE, Finlayson EV, et al. Hospital volume and surgical mortality in the United States. N Engl J Med. 2002; 346:1128-37. [PubMed: 11948273]

8. Sampliner RE, Fennerty B, Garewal HS. Reversal of Barrett's esophagus with acid suppression and multipolar electrocoagulation: preliminary results. Gastrointest Endosc. 1996; 44:532-5. [PubMed: 8934157]

9. Pech O, Behrens A, May A, et al. Long-term results and risk factor analysis for recurrence after curative endoscopic therapy in 349 patients with high-grade intraepithelial neoplasia and mucosal adenocarcinoma in Barrett's oesophagus. Gut. 2008; 57:1200-6. [PubMed: 18460553]

10. Johnston MH, Eastone JA, Horwhat JD, et al. Cryoablation of Barrett's esophagus: a pilot study. Gastrointest Endosc. 2005; 62:842-8. [PubMed: 16301023]

11. Greenwald BD, Dumot JA, Horwhat JD, et al. Safety, tolerability, and efficacy of endoscopic lowpressure liquid nitrogen spray cryotherapy in the esophagus. Dis Esophagus. Jun 9.2009 [Epub ahead of print].

12. Montgomery E, Bronner MP, Goldblum JR, et al. Reproducibility of the diagnosis of dysplasia in Barrett esophagus: a reaffirmation. Hum Pathol. 2001; 32:368-78. [PubMed: 11331953]

13. Canto MI, Gorospe EC, Shin EJ, et al. Carbon dioxide (CO2) cyotherapy is a safe and effective treatment of Barrett's esophagus (BE) with HGD/intramucosal carcinoma [abstract]. Gastrointest Endosc. 2009; 69:AB341.

14. Dumot JA, Vargo JJ, Falk GW, et al. An open-label, prospective trial of cryospray ablation for Barrett's esophagus high-grade dysplasia and early esophageal cancer in high-risk patients. Gastrointest Endosc. 2009; 70:635-44. [PubMed: 19559428]

15. Overholt BF, Panjehpour M, Haydek JM. Photodynamic therapy for Barrett's esophagus: followup in 100 patients. Gastrointest Endosc. 1999; 49:1-7. [PubMed: 9869715] 
16. Sharma VK, Wang KK, Overholt BF, et al. Balloon-based, circumferential, endoscopic radiofrequency ablation of Barrett's esophagus: 1-year follow-up of 100 patients. Gastrointest Endosc. 2007; 65:185-95. [PubMed: 17258973]

17. Ganz RA, Overholt BF, Sharma VK, et al. Circumferential ablation of Barrett's esophagus that contains high-grade dysplasia: a U.S. multicenter registry. Gastrointest Endosc. 2008; 68:35-40. [PubMed: 18355819]

18. Shaheen NJ, Spechler SJ. Total endoscopic eradication of Barrett's esophagus: study methodology, candidate selection, and clinical outcomes. Endoscopy. 2008; 40:994-9. [PubMed: 19065482] 


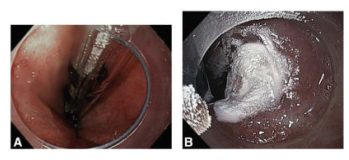

Figure 1.

A, Dual-channel decompression tube in place, as seen through the endoscope with a frictionfit cap before administration of liquid nitrogen. B, White frost demonstrated in the distal esophagus, signaling the freeze time. 


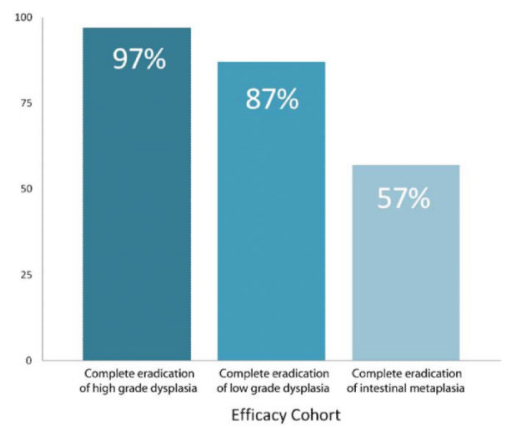

Figure 2.

Efficacy cohort analysis: percentage achieving complete eradication. 


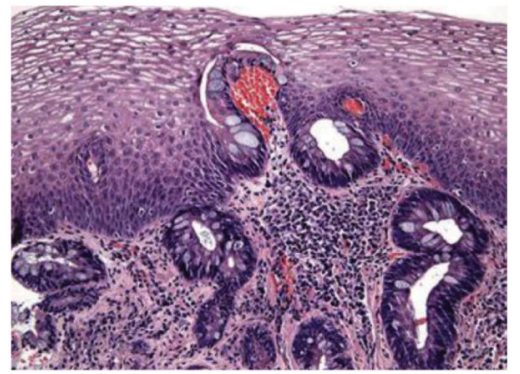

Figure 3.

Subsquamous intestinal metaplasia. 


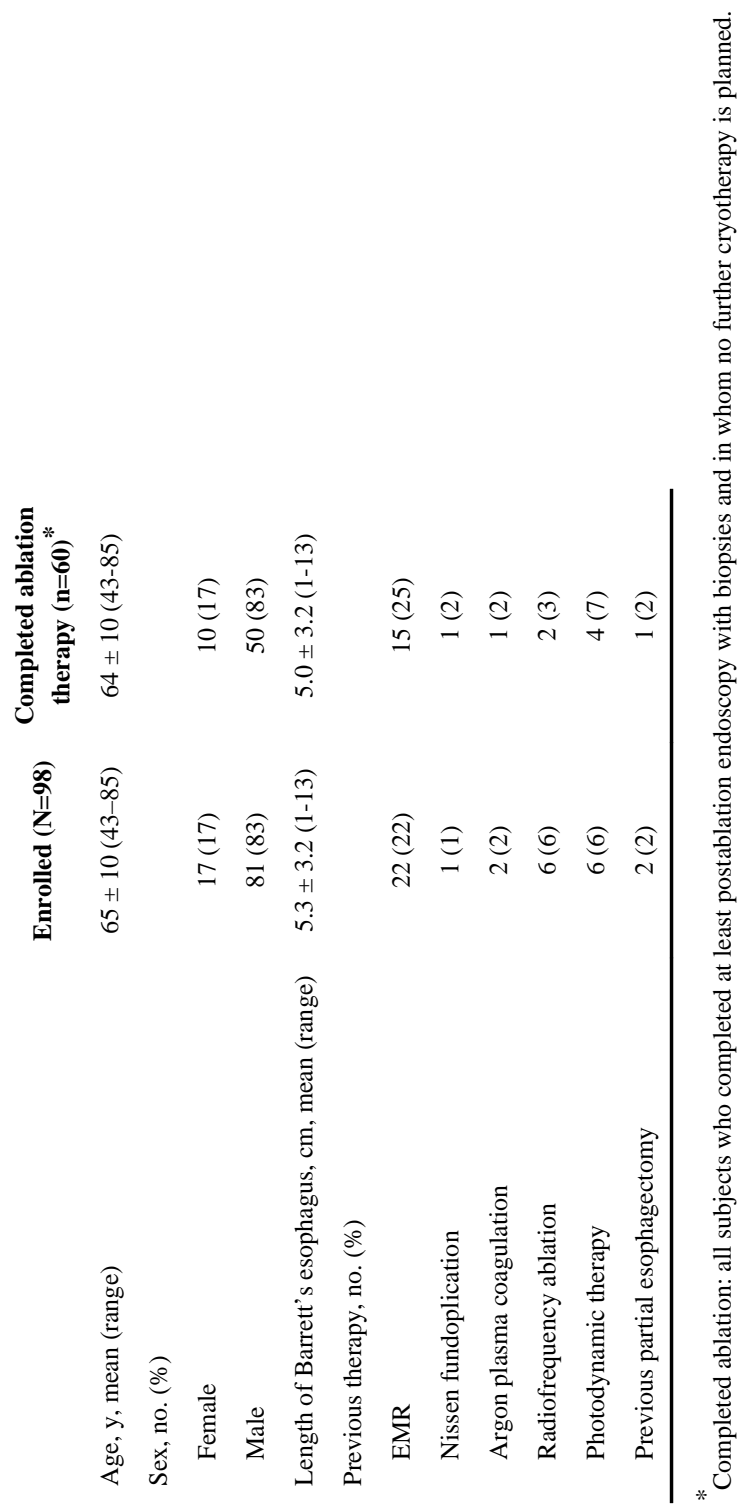

Gastrointest Endosc. Author manuscript; available in PMC 2011 May 13. 
TABLE 2

Procedure variables and adverse events

\begin{tabular}{lc} 
Total no. treatments & $\mathbf{3 3 3}$ \\
${\text { Perforation, no. }(\%)^{*}}^{*} 0(0)$ \\
${\text { Stricture, no. }(\%)^{*}}^{*}$ & $3(3)$ \\
Severe pain, no. $(\%)^{*}$ & $2(2)$ \\
Hospital admission, no. $(\%)^{*}$ & $1(1)$ \\
Mean procedure time, min & $31 \pm 16$ \\
Sedation & \\
\multicolumn{2}{l}{ Propofol } \\
$\quad$ Narcotic/benzodiazepine \\
\hline Percentages are per patient.
\end{tabular}

